\title{
Análisis de las Habilidades Emocionales para el Emprendimiento de PYMES en la Provincia de El Oro
}

\section{Analysis of Emotional Skills for Entrepreneurship in the Province of El Oro}

Iddar Ivan Jaya Pineda

https://orcid.org/0000-0003-3022-9730

María Eugenia Palomeque Solano

https://orcid.org/0000-0002-5636-4829

Ernesto Felipe Novillo Maldonado

https://orcid.org/0000-0002-7778-729X

Luis Fernando Cedillo Chalaco

https://orcid.org/0000-0002-3142-4485

Universidad Técnica de Machala, Ecuador

Autor para correspondencia: ijaya@utmachala.edu.ec; mpalomeque@utmachala.edu.ec; enovillo@utmachala.edu.ec; luiscedillo_analysis@hotmail.com

Fecha de recepción: 24 de marzo del 2020 - Fecha de aceptación: 27 de junio del 2020

\section{Resumen}

La investigación tuvo como objetivo analizar la relación entre las habilidades emocionales y el emprendimiento de administradores de pymes. Para este trabajo usaron un enfoque de tipo cuantitativo aplicado a un grupo de emprendedores de pymes en la provincia de El Oro, cuyos resultados fueron tabulados y analizados mediante la técnica multivariante del análisis factorial y correlacional, cuyos resultados fueron valores superiores a 0,9 , lo que indico fiabilidad y consistencia óptima en los instrumentos; por otro lado en el análisis correlacional, dieron como resultado valores que indicaron que las categorías en su totalidad presentaron correlaciones estadísticamente significativas, en consecuencia quedo demostrada la relación existente entre las categorías usadas en la investigación, en este caso categorías que correspondieron a las habilidades emocionales y el emprendimiento. Por todo esto, resulta necesario que las habilidades emocionales que fueron consideradas en el estudio, no sean atributos aislados en los emprendedores, sino más bien, se cosechen simultáneamente en ellos cada una de las habilidades que explican fuertemente la acción de emprender.

Palabras clave: habilidades emocionales; emprendimiento; emprendedores

\begin{abstract}
The research aimed to analyze the relationship between emotional skills and entrepreneurship of SME managers. For this work, use a quantitative type approach applied to a group of SME entrepreneurs in the province of El Oro, whose results were tabulated and analyzed using the multivariate technique of factorial and correlational analysis, whose results were values greater than 0.9, what indicate precise and optimal consistency in the instruments; On the other hand, in
\end{abstract}


the correlational analysis, it obtained as a result the values that indicate that the categories as a whole, the statistically affected correlations, consequently, that demonstrated the relationship between the categories used in the research, in this case, the categories that correspond to emotional skills and entrepreneurship. For all this, it is necessary that the emotional skills that were considered in the study are not specific attributes in entrepreneurs, but rather, they are simultaneously harvested in each of the skills that strongly explain the action of entrepreneurship.

Key words: emotional skills; entrepreneurship; entrepreneurs

\section{Introducción}

El emprendimiento en la actualidad ha sido de gran interés para los investigadores por su importancia con el progreso y evolución económica de los países. En los mercados actuales se tiene que los empresarios se convierten en emprendedores para lograr que sus empresas vayan adaptándose a las situaciones cambiantes que se presentan en los mercados. Por este motivo adquiere mayor relevancia el emprendimiento para las pequeñas y medianas empresas (PYME), debido a que el emprendimiento le permite ser competitiva.

Los emprendedores deben tener ciertas capacidades y valores necesarios para tener éxito. Formar una empresa es el sueño inicial que tiene un emprendedor, buscando innovar y mejorar los productos y el mercado. Aunque no todos los emprendedores logran tener éxito con sus ideas, luchan por ellas, asumiendo riesgos y sacando adelante sus propuestas (Recalde, Villota, \& Flores, 2016).

La Inteligencia Emocional tiene tres habilidades emocionales necesarias como son: la percepción, la integración y la regulación emocional. El desarrollo de estas habilidades permite el perfeccionamiento de la competencia emocional de un individuo, dichas competencias permiten manejar de mejor manera los acontecimientos vividos, relacionando a un mejor nivel de bienestar de niños y adultos. Así como la capacidad para descubrir, entender, entender y regular las propias emociones (Peñalva, López-Goñi, \& Barrientos, 2017).

Un estudio examinó la relación existente entre la intención emprendedora y algunas variables cognitivas y socio personales, en dicho estudio no se analizó a profundidad las relaciones entre la actitud de emprender y las emociones, no estaban dentro de las hipótesis planteadas; de dichas investigación se impulsó una exigencia para futuras investigaciones para analizar la relación entre la intención emprendedora y los aspectos psicológicos del individuo (Durán-Aponte \& Arias-Gómez, 2015).

La actual investigación posee el objetivo de analizar la relación entre las habilidades emocionales y el emprendimiento de administradores de pymes. La importancia de este trabajo se da por el valor que tiene las pymes en el avance de la economía y en la sociedad (Taborda, Nova, \& Bohórquez, 2018), y al estudiar las distintas relaciones existentes permiten conocer las características que deberían tener y desarrollar para poder emprender con éxito (Torralba, Pérez, \& Martínez, 2017). 


\section{Desarrollo}

\section{Emprendimiento}

Una definición de lo que es emprendimiento involucra en las actitudes la creatividad, a la innovación, el saber tomar la iniciativa, la administración de proyectos personales y profesionales, y la asumir riesgos y la toma de riesgos para conseguir propósitos definidos (Oliver, Galiana, \& Gutiérrez-Benet, 2016). Por su lado la palabra emprendimiento procede de la palabra en francés "entrepreneur" que significa pionero y que representa a la facultad que tiene un individuo de efectuar un esfuerzo añadido para conseguir un determinado resultado, a su vez palabra usada para aquella persona que inicia un nuevo proyecto o empresa (Jaramillo, 2008).

El emprendimiento es una manera de pensar, deducir y proceder encaminada a generar riquezas, siempre buscando aprovechar las distintas oportunidades que se presentan, visto desde una perspectiva global y sostenida a través de un liderazgo adecuado; es asumir riesgos llevados de la manera adecuada para lograr generar valor a la organización, a la situación económica y a la colectividad (Chirinos, Meriño, \& Martínez, 2018).

En la investigación titulada "Intención emprendedora en estudiantes de la Licenciatura de Administración en una Institución de Educación Superior", cuyo objetivo fue establecer si los factores demográficos y el avance académico tienen una influencia de la intención emprendedora, donde a través de una investigación de tipo cuantitativa a estudiantes universitarios de diferentes edades y cuatrimestres, analizando diez dimensiones de la intención de emprendimiento, teniendo como resultados los elementos que influyen en el emprendimiento. Se pudo observar que variables como género, ciudad y estado civil no influyen significativamente en la intención emprendedora (Arteaga, Mexicano, \& González, 2016).

\section{Habilidades emocionales}

Asimismo, otro aspecto que se ha empezado a estudiar por el impacto que este concibe en el desarrollo de los individuos, y a su vez al desarrollo económico de los pueblos son las habilidades emocionales. La habilidad emocional es un componente fundamental para la prevención, el adelanto social y personal, y una habilidad primordial para la vida (Bisquerra, 2003). Por este motivo se consideró relevante para esta investigación la relación que tiene con el emprendimiento.

Existe estudios como la investigación denominada "Evaluación de habilidades no cognitivas para la administración de la educación”, cuyo objetivo es conocer la importancia que tiene las habilidades no cognitivas en el sector educación, la metodología usada fue de tipo cualitativo. Dentro de los resultados se pudo obtener que no existe consenso en los académicos sobre la definición de las habilidades no cognitivas. Pero si se pudo obtener una clasificación de dichas habilidades. La importancia de desarrollar las habilidades no cognitivas permiten que los estudiantes estén más capacitados para adecuarse a la comunidad a las oportunidades de trabajo que se les pudiesen presentar (Higuera \& Lega, 2019). 
La inteligencia emocional se basa en: comprender las emociones de sí mismo, utilizar sus propias emociones, automotivación, e identificar las emociones del resto (Goleman, 1997). Para este estudio se considera los siguientes aspectos: (a) proactividad, (b) ética profesional, (c) empatía, (d) innovación, autonomía y (e) toma de riesgo.

\section{Proactividad}

Proactividad tiene que ver con el cuestionamiento de las personas por qué de las cosas, el qué, el cómo, si hay alguna mejor forma de hacerlo. El ser proactivo es buscar mejor forma de hacer las cosas, con mayor autoestima, más autoconfianza, pero de una manera controlada y lógica, teniendo en consideración varias opciones y alternativas que elegir (Ares, 2004).

\section{Ética profesional}

Entre tantas definiciones sobre ética profesional se considera a la que se refiere a aquellos principios y valores que se basan en juicios sobre el ser humano y la vida que encaminan a las sociedades, convirtiéndose en necesaria para la gestión de las organizaciones en la actualidad (Ricardo \& Rodriguez, 2017).

\section{Empatía}

La palabra empatía fue usada formalmente en el siglo XVIII a través de la palabra "Einfülung", la misma tiene la traducción "sentirse dentro de". Posteriormente en 1909 el término "empatía" fue usado regularmente como la cualidad de sentirse dentro. Existen varias definiciones de empatía, una que se ha considerado en siglo XX es entender lo que piensan los demás (Fernández-Pinto, López-Pérez, \& Márquez, 2008).

\section{Innovación}

Las transformaciones profundas y vertiginosas que vienen experimentando nuestras sociedades han hecho que las dos últimas décadas se haya realizado un esfuerzo para poder adaptarse a los cambios en las organizaciones y en los ciudadanos, dichos cambios han permitido adaptarse al entorno en el que se vive (Méndez, 2001). La innovación se ha transformado en una exigencia como respuesta a los cambios que se vienen dando. Se dice que la innovación es una herramienta de los emprendedores que sirve para aprovechar los cambios y convertirlos en oportunidades.

\section{Autonomía}

Se puede entender a la autonomía emocional como una definición que abarca características y partes afines con la autogestión personal, dentro de dichos elementos se tiene a la autoestima, como una actitud positiva que se tiene ante la vida, por otro lado como la capacidad para poder examinar críticamente las conductas de los individuos ante la sociedad, la responsabilidad, la competencia que se tiene para encontrar los recursos necesarios y la autoeficacia emocional (Bisquerra Alzina \& Pérez Escoda, 2007). 


\section{Toma de riesgo}

Toma de riesgo es una elección intencional de un individuo para tomar un proceder arriesgado (Portell, Riba, \& Bayés, 1997). Por otro lado aseveran que existen diferencias que se dan en distintas dimensiones en la toma de riesgo, dadas especialmente por diferencias en la valoración que se dé, simultáneamente el proceder continúa de manera constante (Weber \& Hsee, 1998).

Existen estudios sobre la relación entre la inteligencia emocional en el emprendimiento, investigaciones como la efectuada en México, en la que los autores mencionan que se ha centrado en formar emprendedores solo en el aspecto técnico, con formación y apoyo de incubadoras de negocios existentes, pero las Mipymes no siempre son exitosas, eso que no se ha considerado otros aspectos a considerar como la inteligencia emocional de los emprendedores, lo que permitiría examinar las fortalezas y debilidades para nuevos emprendimientos, con lo que se estaría resolviendo una limitante de los problemas del fracaso de las pymes (Hernández, Sanchez, \& Rodríguez, 2017).

Asimismo otra investigación que buscaba la relación entre las emociones y las habilidades emprendedoras en empresarios turísticos en el Puyo, a través de una investigación de tipo cuantitativa dieron como resultados que la inteligencia emocional a través de ciertas dimensiones estudiadas tiene alta significancia con una variable de las habilidades emprendedoras analizadas, con lo que quedó demostrado que las habilidades emprendedoras tienen relación con las emociones de los empresarios del sector turístico en el Puyo (Manosalvas, Chávez, Guevara, \& Manosalvas, 2018). Quedando abierta para futuras investigaciones en otros sectores.

\section{Método}

En términos metodológicos, la presente investigación tiene enfoque cuantitativo con alcance exploratorio (Hernández, Fernández, \& Baptista, 2014) (Jaimes, Márquez, \& Pernía, 2015). La que se ve acompañada de un diseño no experimental de enfoque transaccional (Rojas, 2015), en el que se recabará información en un tiempo determinado al aplicar la escala de emprendimiento de habilidades emocionales, que a posteriori serán tabulados y analizados mediante la técnica multivariante del análisis factorial y correlacional en el software estadístico SPSS.

En lo que respecta a la muestra, esta se conforma de 270 individuos categorizados como emprendedores de pymes dentro de los diversos lugares de la provincia de El Oro, a la que se aplicará de manera probabilística aleatoria simple (Otzen \& Manterola, 2017) un instrumento estandarizado tipo encuesta. El tipo de cálculo de obtención del tamaño muestral se lo realizó usando la fórmula para estimar la proporción poblacional cuando la población es infinita (Londoño, Velez, \& Causado, 2016).

$$
n=\frac{Z^{2} \alpha / 2 p q}{e^{2}}
$$


Donde:

$\mathrm{Z}=$ definido por el nivel de confianza $(\alpha / 2)$,

$\mathrm{p}=$ probabilidad de éxito

$\mathrm{q}=$ probabilidad de fracaso

e= error máximo tolerable de muestreo

Para efectos de precisión, los valores acuñados para la obtención de dicha muestra son: $\mathrm{p}=0,50$; $\mathrm{q}=0,50 ; \mathrm{e}=8 \%$ aproximadamente, y $\mathrm{Z}=1,96$ (Valdivieso, Valdivieso, \& Valdivieso, 2011).

\section{Instrumento: Escala de emprendimiento de habilidades emocionales}

El instrumento fue seleccionado realizando una ardua revisión en las bases teóricas que datan sobre habilidades emocionales para el emprendimiento, en las que se seleccionaron de acuerdo en la experiencia holística de investigadores aplicados, expertos en psicometría y motivadores empresariales preguntas que desarrollan las actitudes emprendedoras medidas desde seis dimensiones de habilidades emocionales, tales como: (a) proactividad, (b) ética profesional, (c) empatía, (d) innovación, autonomía y (e) toma de riesgo (Oliver \& Galiana, 2015).

Dicho instrumento en su etapa inicial se conforma de 18 ítems con una escala de 7 puntos de tipo Likert, en la cual el valor de 1 corresponde a "Totalmente en desacuerdo" y 7 refiere a "Totalmente de acuerdo". Estas preguntas a su vez, convergen en seis dimensiones; conformado por exactamente tres ítems cada una, de la siguiente manera:

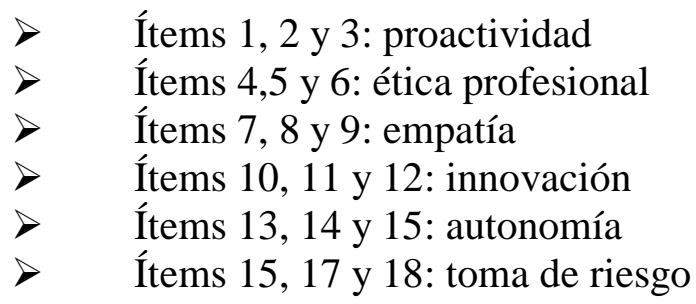

\section{Resultados}

Para que exista confiabilidad dentro de las prácticas investigativas en donde se apliquen instrumentos, es necesario conocer y determinar su nivel de fiabilidad o propiedad de puntuaciones de un test que precisan los resultados obtenidos de este (Ventura \& Caycho, 2017), y para esto surge el coeficiente Alfa de Cronbach, el cual mantiene su nombradía por ser el de mayor uso en estudio psicométricos.

Para lo cual, de acuerdo a lo mostrado en la Tabla 1, el coeficiente de fiabilidad, en el caso de los elementos de forma individual, fue de 0,949 , y coeficiente para las seis dimensiones fue de 0,912; lo que muestra que al ser superior de 0,80 indica una fiabilidad y consistencia óptima (Almanza \& Vargas-Hernández, 2015) (Cedillo \& Campuzano, 2019); es decir que los resultados esperados a posteriori de haber sido aplicado el instrumento son altamente confiables. 
Tabla. 1

Coeficiente de fiabilidad

\begin{tabular}{cc}
\hline Coeficiente & N de elementos \\
\hline 0,949 & 18 \\
0,912 & 6 \\
\hline
\end{tabular}

De acuerdo al análisis correlacional, se aprecia en la Tabla 2 que las categorías en su totalidad presentan correlaciones estadísticamente significativas, valor de p-valué por debajo del 5\% (Barriopedro, 2015) (Ventura, 2017). Estando más altamente correlacionadas, las categorías Proactividad y empatía (79,3\%), seguida con $75,3 \%$ autonomía y toma de riesgos, consecuentemente, ética profesional e innovación con 70,1\%; y aproximadamente con el $70 \%$ de correlación las categorías innovación y autonomía. Por otro lado, entre el $70 \%$ y $60 \%$ de asociación correlacional se encuentran las combinaciones de: empatía-proactividad, empatíaética profesional, innovación-proactividad, innovación empatía, autonomía-proactividad, autonomía-ética profesional, autonomía-empatía, autonomía-innovación; y finalmente (aunque estadísticamente significativas) por debajo del 60\% de correlación toma de riesgo-proactividad, toma de riesgo-ética profesional, toma de riego-empatía y toma de riesgo-innovación.

Tabla. 2

Análisis correlacional

\begin{tabular}{|c|c|c|c|c|c|c|}
\hline & & $\begin{array}{c}\text { Ética } \\
\text { profesional }\end{array}$ & Empatía & Innovación & Autonomía & $\begin{array}{c}\text { Toma } \\
\text { de } \\
\text { riesgo }\end{array}$ \\
\hline \multirow{5}{*}{ Correlación } & Proactividad & 0,793 & 0,665 & 0,645 & 0,599 & 0,471 \\
\hline & Ética profesional & & 0,688 & 0,701 & 0,621 & 0,556 \\
\hline & Empatía & & & 0,667 & 0,613 & 0,51 \\
\hline & Innovación & & & & 0,698 & 0,573 \\
\hline & Autonomía & & & & & 0,753 \\
\hline
\end{tabular}

Para dar paso al análisis factorial ${ }^{1}$, por el método de extracción, se precisa inicialmente el cálculo de las medidas de adecuación muestral, prueba de KMO y además la prueba de esfericidad de Bartlett (Auné \& Attorresi, 2017); la primera toma en cuenta las correlaciones parciales observadas entre variables; y la segunda, evalúa la aplicabilidad de dicho análisis en las variables estudiadas (categorías); las cuales presentan como resultados, para KMO 0,863 que se visualizan en la Tabla 3; y para Bartlett una significancia estadística de 0,000; lo que se traduce, que al ser ambos estadísticos satisfactorios (KMO superior a 0,70 y Bartlett inferior al 5\% ) se puede dar la aplicabilidad del análisis factorial (Gottfried, 2016).

\footnotetext{
1 Modelo estadístico multivariante de reducción de datos que evidencia relaciones que existen entre un grupo de variables que no son observables, o conocidas también como latentes que se denominan factores, que por lo general deben ser menores a las variables originales, debido a la extracción de una varianza común entre estos para formar grupos homogéneos de variables (Fernández, 2015) (García, León, \& Nuño, 2017) (Tirapu-Ustárroz, Cordero, \& Bausela, 2018).
} 
Tabla. 3

Prueba de KMO y Bartlett

\begin{tabular}{llr}
\hline $\begin{array}{l}\text { Prueba de KMO de adecuación de } \\
\text { muestreo }\end{array}$ & $\mathbf{0 , 8 6 3}$ \\
\hline & $\begin{array}{l}\text { Aprox. Chi- } \\
\text { cuadrado }\end{array}$ & 1117,831 \\
Prueba de esfericidad de & Gl & 15 \\
Bartlett & Sig. & 0 \\
\hline
\end{tabular}

Desde el modelo inicial factorial, en la Tabla 4 de comunalidades, se observa que del total de categorías que conforman el test de habilidades emocionales para el emprendimiento; las categorías empatía y toma de riesgo son las peor explicadas por el modelo, en 68,5\% y 58,2\%, respectivamente, desde su variabilidad original, las que se correlacionan entre sí únicamente en un $51 \%$. En cambio, las categorías proactividad, ética profesional, innovación y autonomía son mejor explicadas por el modelo inicial, con valores respectivos de 69,8\%, 76,3\%, 73,5\%, y $72,8 \%$.

Tabla. 4

Comunalidades

\begin{tabular}{llr}
\hline & Inicial & \multicolumn{2}{c}{ Extracción } \\
\hline Proactividad & 1 & 0,698 \\
Ética_profesional & 1 & 0,763 \\
Empatía & 1 & 0,685 \\
Innovación & 1 & 0,735 \\
Autonomía & 1 & 0,728 \\
Toma_de_riesgo & 1 & 0,582 \\
\hline
\end{tabular}

Por consiguiente, al extraer la varianza total explicada dentro del análisis (Tabla 5), se observa que de los componentes extraídos solo el primero, posee un valor por encima de 1 (en lo que refiere a la matriz analizada), el cual consigue explicar en aproximadamente $70 \%$ la varianza de los datos originales; por lo tanto, para que exista la pertinencia de análisis, para este caso únicamente es necesario extraer un solo componente que explicará las habilidades emocionales para el emprendimiento de pymes en la provincia de El Oro, medido desde la (a) proactividad, (b) ética profesional, (c) empatía, (d) innovación, autonomía y (e) toma de riesgo. 
Tabla. 5

Varianza total explicada

\begin{tabular}{ccccccc}
\hline \multirow{2}{*}{ Componente } & \multicolumn{3}{c}{ Autovalores iniciales } & \multicolumn{3}{c}{ Sumas de extracción de cargas al } \\
cuadrado & \multicolumn{2}{c}{$\begin{array}{c}\text { \% de } \\
\text { varianza }\end{array}$} & $\begin{array}{c}\text { \% } \\
\text { acumulado }\end{array}$ \\
& Total & $\begin{array}{c}\text { \% de } \\
\text { varianza }\end{array}$ & $\begin{array}{c}\text { \% } \\
\text { acumulado }\end{array}$ & Total & 69,849 & 69,849 \\
1 & 4,191 & 69,849 & 69,849 & 4,191 & & \\
2 & 0,695 & 11,577 & 81,426 & & & \\
3 & 0,369 & 6,151 & 87,577 & & & \\
4 & 0,327 & 5,448 & 93,025 & & & \\
5 & 0,242 & 4,035 & 97,059 & & & \\
\hline 6 & 0,176 & 2,941 & 100 & & & \\
\hline
\end{tabular}

Nota: Método de extracción de componentes principales.

En consecuencia, la matriz de componente, por el método de extracción de componentes principales (Tabla 6), corrobora lo anteriormente mencionado en la varianza total explicada, tanto en los autovalores iniciales como en las sumas de extracción de cargas al cuadrado, que la solución factorial entre las variables tomadas en cada una de las categorías de la escala de emprendimiento están recogidas en un único componente, al que se le denomina habilidades emocionales para el emprendimiento. Lo que significa, que para que el emprendimiento de pymes en el marco de la provincia de El Oro sea adecuado, es necesario que las habilidades emocionales consideradas en este estudio (categorías), no sean atributos aislados en los emprendedores, sino más bien, se cosechen simultáneamente en ellos cada una de estas habilidades que explican fuertemente la acción de emprender, que van de valores que explican la varianza fuertemente con $76,3 \%$ en lo que respecta a toma de riesgos, $82,8 \%$ de empatía, 83,6\% de proactividad, $85,3 \%$ de autonomía, $85,7 \%$ de innovación , y 87,3\% de ética profesional.

\section{Tabla. 6}

Matriz de componente ${ }^{a}$

\begin{tabular}{lc}
\hline & Componente \\
\hline Proactividad & $\mathbf{1}$ \\
Ética_profesional & 0,836 \\
Empatía & 0,873 \\
Innovación & 0,828 \\
Autonomía & 0,857 \\
Toma_de_riesgo & 0,853 \\
\hline
\end{tabular}

Nota: a. 1 componentes extraídos. 


\section{Conclusiones}

Como consecuencia de la investigación los datos indican que existe relación entre las habilidades emocionales y el emprendimiento de administradores de pymes de la provincia de El Oro, para la cual se alcanza un Alfa de Cronbach de 0,912 demostrando la fiabilidad del instrumento usado para la investigación. Posterior a esto se tienen los resultados de la investigación a través de un análisis correlacional, que implica que las categorías en su totalidad presentan correlaciones estadísticamente significativas, en consecuencia, queda demostrada la relación existente entre las categorías usadas en la presente investigación, en este caso categorías que corresponden a las habilidades emocionales y el emprendimiento.

Los resultados de la solución factorial demuestran que para que el emprendimiento de pymes en el entorno de la provincia de El Oro sea adecuado, resulta necesario que las habilidades emocionales consideradas en el estudio, no sean atributos aislados en los emprendedores, sino más bien, se cosechen simultáneamente en ellos cada una de estas habilidades que explican fuertemente la acción de emprender.

La investigación abre las puertas al análisis del emprendimiento tomando en consideración distintas variables que permitirán conocer donde deben trabajar las instituciones de Educación Superior y el Gobierno para promover el emprendimiento en los profesionales ya que este ayudara al avance económico y social de la sociedad.

\section{Bibliografía}

Almanza, R., \& Vargas-Hernández, J. (2015). Las Competencias Profesionales y su relación con la empleabilidad de los Ingenieros en Gestión Empresarial egresados del ITLAC. Revista Electrónica Gestión de las Personas y Tecnología, 8(22), 17-28. doi:https://www.redalyc.org/articulo.oa?id=4778/477847104002

Ares, A. (2004). La conducta proactiva de los emprendedores. Portularia, 4, 493-498. Obtenido de http://rabida.uhu.es/dspace/bitstream/handle/10272/179/b15139347.pdf?sequenc

Arteaga, A., Mexicano, M., \& González, R. (2016). Intención emprendedora en estudiantes de la Licenciatura de Administración en una Institución de Educación Superior. XXI Congreso Internacional de Contaduría, Administración e Informática, (págs. 1-19). México. Obtenido de http://congreso.investiga.fca.unam.mx/docs/xxi/docs/8.03.pdf

Auné, S., \& Attorresi, H. (2017). Dimensionalidad de un Test de Conducta Prosocial. Revista Evaluar, 17(1), 29-37. doi:https://doi.org/10.35670/1667-4545.v17.n1.17072

Barriopedro, M. (2015). La significación estadística no es suficiente. [Statistical significance is not enough]. Revista Internacional de Ciencias del Deporte, 11(40), 101-103. doi:http://dx.doi.org/10.5232/ricyde2015.040ed

Bisquerra Alzina, R., \& Pérez Escoda, N. (2007). Las competencias emocionales. Educación, 21(10), 61-82. doi:10.5944/educxx1.1.10.297

Bisquerra, R. (2003). Educación emocional y competencias básicas para la vida. Revista de Investigación Educativa, 21(1), 7-43. Obtenido de https://revistas.um.es/rie/article/view/99071 
Cedillo, L., \& Campuzano, J. (2019). Modelo de probabilidades para el análisis del desempleo en la ciudad de Machala- Ecuador. INNOVA Research Journal, 4(3), 23-37. doi:https://doi.org/10.33890/innova.v4.n3.2019.866

Chirinos, Y., Meriño, V., \& Martínez, C. (2018). El clima organizacional en el emprendimiento sostenible. $\quad$ Revista $\quad$ EAN(84), 43-61. doi:http://dx.doi.org/10.21158/01208160.n84.2018.1916

Durán-Aponte, E., \& Arias-Gómez, D. (2015). Intención emprendedora en estudiantes universitarios: integración de factores cognitivos y socio-personales. Revista Colombiana de Ciencias $\quad$ Sociales, 6(2), 320-340. Obtenido de http://www.funlam.edu.co/revistas/index.php/RCCS/article/view/1528/pdf_21

Fernández, A. (2015). Aplicación del análisis factorial confirmatorio a un modelo de medición del rendimiento académico en lectura. Ciencias Económicas, 33(2), 39-66. doi:10.15517/RCE.V33I2.22216

Fernández-Pinto, I., López-Pérez, B., \& Márquez, M. (2008). Empatía: Medidas, teorías y aplicaciones en revisión. Anales de psicología, 24(2), 284-298. Obtenido de https://revistas.um.es/analesps/article/view/42831

García, J., León, J., \& Nuño, J. (2017). Propuesta de un modelo de medición de la competitividad mediante análisis factorial. Contaduría y Administración, 62(3), 775-791. doi:https://doi.org/10.1016/j.cya.2017.04.003

Goleman, D. (1997). Inteligencia emocional. Barcelona: Kairós.

Gottfried, A. (2016). Adaptación Argentina del PIL Test (Test de Sentido en la Vida) de Crumbaugh y Maholick. Revista de Psicología, 12(23), 49-65. Obtenido de http://200.16.86.39/index.php/RPSI/article/view/1536/1452

Hernández, N., Sanchez, R., \& Rodríguez, R. (2017). La inteligencia emocional en el emprendimiento: una discusión obligada para generar emprendedores exitosos. Caderno Profissional de Marketing-UNIMEP, 5(2), 39-51. Obtenido de https://www.cadernomarketingunimep.com.br/ojs/index.php/cadprofmkt/article/view/104 183

Hernández, R., Fernández, C., \& Baptista, M. d. (2014). Metodología de la investigación (Sexta edición ed.). México D. F.: McGraw Hill.

Higuera, S., \& Lega, M. (2019). Evaluación de habilidades no cognitivas para la administración de la educación. Revista Estrategia Organizacional, 8(2), 103-144. doi:https://doi.org/10.22490/25392786.3434

Jaimes, S., Márquez, J., \& Pernía, L. (2015). Factores psicosociales que influyen en el comportamiento laboral de acuerdo con los procesos de gestión administrativa y del talento humano que presentan los empleados de a empresa Distraves S.A de Cúcuta. Revista Mundo FESC, 5(10), 23-26. Obtenido de https://www.fesc.edu.co/Revistas/OJS/index.php/mundofesc/article/view/73

Jaramillo, L. (2008). Emprendimiento: Concepto básico en competencias. Lumen. Revista del Instituto de Estudios en Educación-IESE, 7, 1-6. Obtenido de https://guayacan.uninorte.edu.co/divisiones/iese/lumen/ediciones/7/index.html

Londoño, A., Velez, O., \& Causado, L. (2016). Selección de mercados extranjeros en empresas PYME del sector del plástico en Medellín, Colombia. Espacios, 37(2), 3-14. Obtenido de https://www.revistaespacios.com/a16v37n02/16370203.html

Manosalvas, L., Chávez, G., Guevara, D., \& Manosalvas, C. (2018). Influencia de las emociones en las habilidades emprendedoras de los propietarios de servicios turísticos de la ciudad de 
Puyo. Killkana sociales: Revista de Investigación Científica, 2(3), 87-94. doi:https://doi.org/10.26871/killkana_social.v2i3.327

Méndez, R. (2001). Innovación y redes de cooperación para el Desarrollo Local. Revista Internacional de Desenvolvimento Local, 2(3), 37-44. doi:https://doi.org/10.20435/interações.v2i3.585

Oliver, A., \& Galiana, L. (2015). Development and Validation of the Escala de Actitudes Emprendedoras para Estudiantes (EAEE). The Spanish Journal of Psychology, 18, 1-10. doi:https://doi.org/10.1017/sjp.2015.14

Oliver, A., Galiana, L., \& Gutiérrez-Benet, M. (2016). Diagnóstico y políticas de promoción del emprendimiento en estudiantes. Anales de Psicología/Annals of Psychology, 32(1), 183 189. doi:https://doi.org/10.6018/analesps.32.1.186681

Otzen, T., \& Manterola, C. (2017). Técnicas de muestreo sobre una población a estudio. International Journal of Morphology, 35(1), 227-232. doi:http://dx.doi.org/10.4067/S071795022017000100037

Peñalva, A., López-Goñi, J., \& Barrientos, J. (2017). Habilidades emocionales y profesionalización docente para la educación inclusiva en la sociedad en red. Contextos Educativos. Revista de Educación(20), 201-215. doi:http://doi.org/10.18172/con.3011

Portell, M., Riba, M. D., \& Bayés, R. (1997). La definición de riesgo: implicaciones para su reducción. Revista de Psicología de la Salud, 9(1), 3-27. doi:https://doi.org/10.21134/pssa.v9i1.1195

Recalde, L., Villota, L., \& Flores, M. (2016). Emprendedores como creadores de riqueza y desarrollo regional. Revista Publicando, 3(9), 564-578. Obtenido de https://dialnet.unirioja.es/descarga/articulo/5852122.pdf

Ricardo, C., \& Rodriguez, R. (2017). Responsabilidad social y ética profesional en la gestión de la administración pública y empresarial. Pensamiento \& Gestión(42), 6-25. doi:http://dx.doi.org/10.14482/pege.42.10452

Rojas, M. (2015). Tipos de Investigación científica: Una simplificación de la complicada incoherente nomenclatura y clasificación. Redvet, 16(1), 1-14. Obtenido de https://www.redalyc.org/pdf/636/63638739004.pdf

Taborda , M., Nova, L., \& Bohórquez, I. (2018). Importancia de las Pymes para el desarrollo económico de Chile en el siglo XXI. Punto de vista, 9(14), 1-18.

Tirapu-Ustárroz, J., Cordero, P., \& Bausela, E. (2018). Funciones ejecutivas en población infantil: propuesta de una clarificación conceptual e integradora basada en resultado de análisis factoriales. Cuadernos de Neuropsicología, 12(3), 1-31. doi:10.7714/CNPS/12.3.203

Torralba, A., Pérez, A., \& Martínez, D. (2017). Fracaso emprendedor, como experiencia de aprendizaje para empresarios del municipio de Puebla. Revista GEON, 4(2), 25-33. doi:https://doi.org/10.22579/23463910.21

Valdivieso, C., Valdivieso, R., \& Valdivieso, O. (2011). Determinación del tamaño muestral mediante el uso de árboles de decisión. UPB - Investigación \& desarrollo, 1(11), 148-176. Obtenido de http://www.upb.edu/revista-investigaciondesarrollo/index.php/id/article/view/64/188

Ventura, J. (2017). El significado de la significancia estadística: comentarios a Martínez-Ferrer y colaboradores. Salud pública Méx, 59(5), 499-500. doi:http://dx.doi.org/10.21149/8482

Ventura, J., \& Caycho, T. (2017). El coeficiente Omega: un método alternativo para la estimación de la confiabilidad. Revista Latinoamericana de Ciencias Sociales, Niñez y Juventud, 15(1), 625-627. Obtenido de https://www.redalyc.org/pdf/773/77349627039.pdf 
Weber, U., \& Hsee, C. (1998). Cross-cultural differences in risk perception but, cross-cultural similarities in attitudes towards risk. Management Science, 44(9), 1205-1217. doi:https://doi.org/10.1287/mnsc.44.9.1205 